\title{
Ingenia sanare: Die Therapie der Undankbarkeit in Senecas De beneficiis
}

\author{
Antje Junghanß \\ (Dresden University of Technology) \\ Ingenia sanare. Seneca's Therapy of Ingratitude in De beneficiis
}

\begin{abstract}
For Seneca (and the Stoics in general) benefactions are the essential element for social bonding: they are meant to be reciprocal and thus create relationships. But as he points out in the beginning of De beneficiis they often remain unanswered (and thus fail to create or stabilize community life) because of the recipients' ingratitude. What Seneca undertakes in De beneficiis is to teach people how to avoid ingratitude. As he points out in the beginning of the treatise, a real philosopher's duty is to heal people's souls, so his approach is a therapeutic one. In my paper I present Seneca's therapy of ingratitude. I argue that it is rooted in Stoic Ethics and at the same time adapted to his non-sage readers.
\end{abstract}

\section{Keywords}

Stoic ethics; therapy; benefactions; ingratitude 
Im Prooem seiner siebenbändigen Schrift De beneficiis spottet Seneca über den Hang griechischer Stoiker, Nutzloses zu erörtern, ${ }^{1}$ obwohl sie doch eigentlich Wesentliches zu lehren hätten, nämlich: ein Gesetz des Lebens zu formulieren, das zu einem rechten Umgang mit Wohltaten befähige. Wohltaten sind für Seneca diejenige Sache, welche die Menschengemeinschaft in besonderer Weise (maxime) verbindet (adligat). ${ }^{2}$ Anstatt sich bei Banalitäten (ineptiae) aufzuhalten, die eigentlich den Dichtern überlassen bleiben sollten, sei es die Pflicht wahrer Philosophen, ernsthaft zu sprechen (serio loquantur) und sich alle Mühe zu geben (magnis viribus agant), um das zu erreichen, wozu sie angetreten seien: die Menschen in ihrem Wesen zu heilen (ingenia sanare [...] volunt) und für die Bemeisterung des Lebens zu befähigen. ${ }^{3}$

Dem Abschnitt lässt sich entnehmen, dass für Seneca (der sich - selbstredend - den wahren Philosophen zurechnet) die entscheidenden Fragen der Philosophie aus dem Bereich der Ethik stammen und insbesondere (lex vitae) das gemeinschaftliche Miteinander in den Blick nehmen müssen. Gemeinschaft wird in erster Linie durch den Austausch von Wohltaten gestiftet und gestärkt; ${ }^{4}$ Wohltaten haben verbindende Kraft. ${ }^{5}$ Recht verstandenes Wohltun bedarf der Anleitung (doce), scheint sich also nicht von selbst zu ergeben, sondern im Gegenteil häufig fehlerbehaftet zu sein. Diese Fehler wurzeln offenbar in einer inneren (ingenia) Fehlhaltung, die zu behandeln ist (sanare).

Vokabeln aus dem Wortfeld von Heilung und Behandlung zieht Seneca in De beneficiis immer wieder heran. ${ }^{6}$ Raccanelli formuliert, das Werk „assume i connotati di una tera-

1 Cf. benef. 1,3,6-10.

2 ... res, quae maxime humanam societatem adligat. (benef. 1,4,2).

3 Tu me aliquid eorum doce, per quae beneficentior gratiorque aduersus bene merentes fiam, per quae obligantium obligatorumque animi certent, ut, qui praestiterunt, obliuiscantur, pertinax sit memoria debentium. Istae uero ineptiae poetis relinquantur, quibus aures oblectare propositum est et dulcem fabulam nectere. At qui ingenia sanare et fidem in rebus humanis retinere, memoriam officiorum ingerere animis uolunt, serio loquantur et magnis uiribus agant... (benef. 1,4,5 et seq.).

4 „Wohltat“ ist im weitestmöglichen Wortsinn zu verstehen und auf alle Handlungen zu beziehen, die abseits vertraglicher Bindungen einem anderen Menschen zum Wohl gereichen sollen. Wohltaten können materielle Zuwendungen aller Art umfassen, aber auch Rat, Zuspruch, wohlwollende Kritik. Zur gemeinschaftsstiftenden Kraft von Wohltaten u.a. bei Seneca cf. Junghanß (2017: passim).

5 Wie Marcel Mauss in seinem berühmten „Essai sur le don“ ausführt, galten bereits in archaischer Zeit Gaben als das wesentliche Mittel, um in Verbindung zu anderen Clans zu treten bzw. bereits geknüpfte Verbindungen zu bestätigen. Eine Gabe anzunehmen, ohne sie zu vergelten, war ein schwerer Affront, der beinahe einer Kriegserklärung gleichkam. Wenngleich sich die mauss'schen Beobachtungen nicht grundsätzlich auf das römische beneficium anwenden lassen, liegt doch der Gedanke der Reziprozität von Geben und Nehmen sowie, damit verbunden, die Überlegung der beziehungsstiftenden Kraft wechselseitiger Gaben auch Senecas Ausführungen zugrunde. Seneca illustriert die Bedingung der Wechselseitigkeit mit zwei Bildern, einmal mit dem Reigen der Grazien, die, einander an den Händen haltend, das beständige Ineinandergreifen von Geben, Annehmen und Vergelten symbolisieren. Und außerdem mit einem Ballspiel, wo Werfen, Fangen und Zurückwerfen dauerhaft aufeinander folgen (Reigen: benef. 1,3,2-5; Ballspiel: ab 2,17,3). Reigen und Ballspiel werden unterbrochen, wenn einer der Beteiligten plötzlich nicht mehr mittanzt oder mitspielt.

6 Setaioli (2014a: pp. 239f.) spricht von einer „medical metaphor“, die Senecas philosophisches Werk durchziehe. In den philosophischen Schriften verwendet Seneca allein sanitas/sanare/sanus 80 mal (Delatte \& Evrard 1981). 
pia, in base all'immagine della filosofia come medicina dell'anima“. ${ }^{7}$ Die Vorstellung, dass philosophische (näherhin ethische) Anleitung als Therapie gelten kann, die auf eine Heilung der Seele abzielt, ist durchaus gängig. ${ }^{8}$ Wenn nun Seneca einen solchen therapeutischen Anspruch an den Anfang seiner Auseinandersetzung mit dem beneficium setzt, lässt sich fragen, wie im Bezug auf dieses konkrete Sujet eine Therapie zu denken ist. Dieser Frage sind die folgenden Betrachtungen gewidmet. Um Senecas therapeutischem Ansatz auf die Spur zu kommen, wollen wir diejenigen Passagen des Werkes in den Blick nehmen, in denen er fehlerhaftes Verhalten beschreibt und Ratschläge zu dessen Verbesserung formuliert. ${ }^{9}$

Fehlverhalten lässt sich stets daran erkennen, dass der Empfänger einer Wohltat undankbar reagiert und die Wohltat damit unerwidert (also: unvergolten) bleibt. Undankbarkeit ist für Seneca ein weit verbreitetes Übel ${ }^{10}$ welches das Gedeihen der Gemeinschaft in Gefahr bringt, insofern als es dazu führt, dass Wohltaten nicht verbindend wirken können. Senecas Therapie muss somit auf eine Heilung von der Undankbarkeit gerichtet sein. ${ }^{11}$ Im zweiten Buch De beneficiis fragt Seneca, was einen Menschen eigentlich undankbar mache, und zählt eine Reihe von Fehlhaltungen auf - Selbstüberschätzung, Habgier, Ehrgeiz und Neid -, denen gemeinsam sei, dass sie keine Zufriedenheit mit empfangenen beneficia erlauben: Wer sich selbst überschätze, beanspruche bei anderen stets den ersten Rang und neige rasch dazu, sich über eine gefühlte Zurücksetzung zu ärgern. ${ }^{12}$ Habgier und Ehrgeiz führten dazu, das Empfangene stets zum Anlass zu nehmen, noch mehr und noch Größeres zu wünschen. ${ }^{13}$ Der Neid schließlich bewirke, dass man die eigene Situation beständig mit der anderer vergleiche. ${ }^{14}$

Senecas Rat an die Adresse derer, die ihrem Eindruck nach zu wenig Wertschätzung erfahren, geht aus von der Überlegung, dass niemand anders sie so sehr ehren könne

7 Raccanelli (2009: p. 304). Allerdings wird bei ihr der Gedanke nicht weiter spezifiziert und spielt auch in ihrer Argumentation keine Rolle. Ferner Long (2003: p. 192); Abel (1995: p. 45).

8 Im HWPh wird unter dem Lemma „Therapie“ vermerkt, dass darunter eigentlich eine Achtungsbezeugung bzw. ein Dienst gegenüber den Göttern, dann aber auch die Pflege Alter, Kranker und Kinder zu verstehen sei. Therapie als Heilung der Seele „werde seit alters her als eine Aufgabe des Philosophen und des Weisen angesehen“. Cf. hierzu vor allem auch Hadot (1991: p. 15, ähnlich pp. 14 und 16), für den antike Philosophie „in erster Linie als Therapie der Leidenschaften“ erscheint.

9 Im APA Dictionary of Psychology (VandenBos 2007: p. 937), wird Therapie definiert als eine „remediation of psychical, mental, or behavioral disorders or disease“. Seneca setzt bei behavioral disorders an, die an sich zugleich der Indikator einer inneren Unordnung sind. Ich möchte betonen, dass ich Senecas Ansatz nicht in Relation zu modernen Psychotherapien setze, sondern allein aus seiner bzw. der stoischen Lehre heraus vorstelle.

10 Nec mirum est inter plurima maximaque vitia nullum esse frequentius quam ingrati animi. (benef. 1,1,2).

11 ... nihil aeque concordiam humani generis dissociat ac distrahit quam hoc vitium. (benef. 4,18,1).

12 Nemo non benignus est sui iudex; inde est, ut omnia meruisse se existimet et in solutum accipiat nec satis suo pro pretio se aestimatum putet. (benef. 2,26,2).

13 Non patitur aviditas quemquam esse gratum; numquam enim inprobae spei, quod datur, satis est [...]. Aeque ambitio non patitur quemquam in ea mensura honorum conquiescere, quae quondam eius fuit inpudens votum. (benef. $2,27,3$ et seq.).

14 Omnibus his vehementius et inportunius malum est invidia, quae nos inquietat, dum conparat. (benef. 2,28,1). 
wie sie selbst. ${ }^{15}$ Das heißt, wer anderen mit der Erwartungshaltung begegne, sich von ihnen in besonderer Weise ausgezeichnet zu sehen, müsse darin immer wieder enttäuscht werden, weil die Würdigung von außen nie an den eigenen Anspruch nach Anerkennung heranreichen könne. Dies gelte es sich bewusst zu machen, dann ergebe sich - anstelle der Verärgerung - eine nachsichtige Haltung gegenüber dem anderen: Dann könne man sich sagen, man hätte durchaus mehr verdient, doch der andere sei nicht imstande gewesen, seine Wertschätzung durch eine bedeutendere Gabe auszudrücken (benef. 2,28,2).

Denen, die zu beständigem Vergleichen neigen, rät Seneca, dass sie doch, anstelle darauf zu schauen, wie viel mehr X erhalten hat, sich daran freuen könnten, selbst mehr als Y zu bekommen. Und wenn man feststellen müsse, dass jemand, der einem selbst weder an Vorzügen noch an Leistungen ebenbürtig sei, reicher beschenkt wurde, dann solle man diesen Umstand dem Schicksal zurechnen und sich sagen, dass der andere einfach Glück gehabt hätte. Auch hier werden wieder Vergleiche angestellt, diesmal aber mit dem Ziel, das Empfangene nicht als Zurücksetzung der eigenen Person zu verstehen, sondern es wohlwollend deuten zu können. ${ }^{16}$

Seneca bestimmt in seiner Darstellung noch einen weiteren Typus des Undankbaren, nämlich denjenigen, der eine empfangene Wohltat hastig vergilt, kaum, dass er sie angenommen hat. Der Grund für ein solches Verhalten liegt seiner Ansicht nach darin, dass der Empfänger mit der Schuld nicht gut zurecht kommt, in der er beim anderen dann steht, und sich möglichst schnell daraus befreien will. ${ }^{17}$ Mit der Schuld geht eine - zumindest momentane - Unterlegenheit dem Wohltäter gegenüber einher. ${ }^{18}$ Das Unvermögen, diese Unterlegenheit auszuhalten, kann nach Senecas Darstellung mitunter so weit führen, dass der Empfänger einer Wohltat seinem Wohltäter ein Unheil an den Hals wünscht, von dem er ihn dann befreien kann, um selbst wieder in die überlegene Position zu gelangen. ${ }^{19}$

15 ... scire neminem tanti ab alio, quanti ab se ipso aestimari. (benef. 2,28,1).

16 Nullum est tam plenum beneficium, quod non vellicare malignitas possit, nullum tam angustum, quod non tam bonus interpres extendat (benef. 2,28,4). Die Deutung hängt vom Blickwinkel ab. Sogar die Wohltaten, welche die Götter den Menschen im Übermaß zur Verfügung gestellt haben, meint Seneca, ließen sich schmälern; auch da fänden sich noch Gründe, unzufrieden zu sein (Numquam deerunt causae querendi, si beneficia a deteriore parte spectaveris; benef. 2,28,4). Seneca fordert einen imaginären Gesprächspartner, der die göttlichen Gaben so kritisch und missgünstig beurteilt, auf, dasjenige zu berücksichtigen, was dem Menschen gegeben sei (Proinde, quisquis es inicus aestimator sortis humanae, cogita, quanta nobis tribuerit parens noster; benef. 2,29,4). Cogita, heißt es im Text; die Aufgabe, die sich für den Empfänger einer Wohltat immer stellt, ist, die Gabe in ihrem Kontext zu betrachten und prinzipiell zu einer nachsichtigen Beurteilung des Gebenden bereit zu sein.

17 Expecta! Quare subsidere aput te munus meum non vis? Quare obligatum moleste te fers? [...]. Ipsam hanc cupiditatem primo quoque tempore liberandi se meminerimus ingrati esse... (benef. 6,39,2-6,40,1). Griffin (2013: p. 303), spricht hier von einer „mistaken motivation of such a beneficiary“.

18 Hierzu Därmann (2010: p. 25).

19 Sollicitudinem illi et metum et maius aliquod inprecaris malum. Optas, ut ope indigeat: hoc contra illum est; optas, ut ope tua indigeat: hoc pro te est. [...] Superiorem te facis et, quod nefas est, bene meritum ad pedes tuos mittis. (benef. 6,27,1 et seq.). 
Bei diesem Bestreben, aus der Unterlegenheit herauszukommen, setzt Seneca nun an: Seiner Ansicht nach ist das Vermögen, eine Wohltat zu schulden, ebenso groß wie die Tatsache, sie erwiesen zu haben. Das heißt, wenn der Empfänger eine Wohltat dankbar entgegennimmt und die Gelegenheit zur Vergeltung ruhig abwartet, zeugt das für Seneca von innerer Größe. Wer sich so verhält, ist seinem Wohltäter in der Haltung ebenbürtig und hat also keinen Grund, sich unterlegen zu fühlen. Tatsächlich sei das Empfangen von Wohltaten sogar mühevoller, weil man das, was man bekommen hat, mit größerer Sorgfalt bewache, als es der andere gegeben habe. ${ }^{20}$

Nun kann es für Seneca jedoch auch vorkommen, dass jemand gern bereit wäre, die Gabe eines anderen dankbar entgegen zu nehmen, sein Dank jedoch durch die Haltung des Gebenden gleichsam verdorben wird. Es existierten viele undankbare Menschen, schreibt Seneca, doch noch mehr Menschen mache man erst dazu: ${ }^{21}$ Wer könne denn jemandem dankbar sein, der einem seine Wohltat entweder stolz vor die Füße geworfen, zornig aufgedrängt oder ermüdet erwiesen hat, um nicht weiter belästigt zu werden?22

Nicht immer ist also derjenige, der undankbar reagiert, derjenige, der sich falsch verhält. Auch der Gebende muss, wie Seneca betont, sein Handeln überprüfen, denn: Eine Wohltat werde immer in derselben Gesinnung geschuldet, in der sie erwiesen wurde..$^{23}$ Für Seneca ist es besonders der Hochmut des Gebenden, der die Undankbarkeit beim Empfänger provoziert; Hochmut mache jede Wohltat zu einer Demütigung. ${ }^{24}$ Hochmut begegne, so meint er, vor allem bei denen, die vom Schicksal begünstigt würden; Hochmut sei das Bemühen, die eigene Überlegenheit als Wohltäter besonders herauszustellen. Demjenigen, der sich - aufgrund glücklicher Umstände - dem anderen gegenüber in der überlegenen Position befindet, rät er zur Zurückhaltung: Viel edler sei es doch, sich selbst mit dem, der eigentlich niedriger steht, auf eine Stufe zu begeben und die Wohltat quasi von gleich zu gleich zu erweisen. ${ }^{25}$

In der Zusammenschau ergibt sich, dass im Kampf gegen die Undankbarkeit Fehlhaltungen sowohl des Empfängers als auch des Wohltäters zu korrigieren sind. Doch nun lässt sich fragen, ob Seneca in seinen Ratschlägen den eingangs zitierten Anspruch einlöst, die Menschen in ihrem Wesen zu heilen. Konsequent stoisch gedacht, müsste sein Ansatz dazu verhelfen, diejenigen, die sich falsch verhalten, dazu zu bringen, sich selbst als neidisch, gierig oder geizig zu erkennen und diese Fehlhaltungen abzulegen. ${ }^{26}$ Wie

20 ... non minoris est animi beneficium debere quam dare; eo quidem operosius hoc quam illud, quo maiore diligentia custodiuntur accepta quam dantur. (benef. 6,43,1).

21 Multos experimur ingratos, plures facimus... (benef. 1,1,4).

22 Gratus adversus eum esse quisquam potest, qui beneficium aut superbe abiecit, aut iratus inpegit aut fatigatus, ut molestia careret, dedit? (benef. 1,1,7).

23 Eodem animo beneficium debetur, quo datur. (benef. 1,1,8).

24 O superbia, magnae fortunae stultissimum malum! Ut a te nihil accipere iuvat! Ut omne beneficium in iniuriam convertis! (benef. 2,13,1).

25 Iocunda sunt, quae humana fronte, certe leni placidaque tribuuntur, quae cum daret mihi superior, non exultavit supra me, sed quam potuit benignissimus fuit descenditque in aecum et detraxit muneri suo pompam... (benef. 2,13,2).

26 Nach Hadot (1991: p. 42) ist die stoische Philosophie (wie andere antike Philosophenschulen auch) auf eine „Wandlung und Verwirklichung“ des Menschenwesens gerichtet; sie besteht zu einem Gutteil aus 
Seneca im 50. Brief an Lucilius ausführt, müsse eine Heilung stets von solcher Selbsterkenntnis ausgehen, wenngleich sie, wie er einräumt, schwer auszuhalten sei. ${ }^{27}$

In den beschriebenen Beispielen aus De beneficiis ist nun bemerkenswert, dass die Selbsterkenntnis zunächst darauf beschränkt bleibt, sich selbst als einen Menschen zu begreifen, der eine herausgehobene Position beansprucht. Denn fragt man, welche Haltungen den Darstellungen zugrunde liegen, so lässt sich - etwas verkürzt - sagen, dass dahinter ein hohes Geltungsbedürfnis des jeweils Handelnden greifbar wird. ${ }^{28}$ An diesem Geltungsbedürfnis rüttelt Seneca nicht, wenngleich es - stoisch betrachtet - eigentlich nichtig wäre. Stattdessen bemüht er sich, genau aus diesem Bedürfnis heraus bewusste Entscheidungen für dankbares Verhalten zu ermöglichen: Die hohe Meinung, die jemand von sich selbst hat, kann er weiter vertreten, solange er sich bewusst macht, dass Wohltaten, die er empfängt, nicht die Höhe der Wertschätzung ausdrücken, die er selbst für seine eigene Person empfindet. Das Vergleichen, das den Neidischen kennzeichnet, darf er fortsetzen - nur eben im Bemühen, die empfangene Wohltat möglichst bedeutend werden zu lassen. Die Überlegenheit, die jemand in der Beziehung zu einem anderen beansprucht, bleibt ihm erhalten, wenn er den dankbaren Empfang einer Wohltat als eine der Gabe selbst gleichwertige und sogar noch mühevollere Handlung begreifen kann. Und der Gebende, der im Handeln seine Vorrangstellung markieren will, muss diese nicht aufgeben. Zwar soll er sich mit dem Empfänger auf eine Stufe stellen, doch durch eine solche edle Haltung kann er sich wahrhaft überlegen zeigen.

Anstelle die Fehlhaltungen als solche zu verurteilen, strebt Seneca in den angeführten Beispielen eine Verhaltensmodifikation an, die genau auf diesen Haltungen aufbaut das Verhalten wird zwar in einer Weise korrigiert, dass Undankbarkeit vermieden und ein Ineinandergreifen von Wohltat und Vergeltung möglich wird, die zugrundeliegende Haltung wird aber nicht überprüft. Was Seneca jedoch zugleich betont, ist, dass die Motive eigenen Handelns sorgfältig zu reflektieren sind, denn häufig sei vernunftgemäßes iudicium durch affectus überdeckt. ${ }^{29}$ Seine Ratschläge zielen also durchaus darauf ab, vernunftgesteuert zu handeln - freilich nicht in der Reflexionstiefe, dass die den Affekten zugrundeliegenden Handlungsmotive in ihrer Berechtigung hinterfragt würden.

Zugleich zeigt sich in den besprochenen Beispielen noch Folgendes: Insbesondere in der Aussage, dass wahre Überlegenheit in einer besonders hochherzigen Haltung bestehen könne, verbindet Seneca seinen konzilianten Ansatz, den Geltungs- oder Überlegenheitsanspruch bestimmter Wohltäter bzw. Empfänger hinzunehmen, mit einem

geistigen Übungen, die, wie er ausführlich am Beispiel der „Ermahungen“ des Marc Aurel beschreibt, dazu befähigen sollen, im Einklang mit der eigenen Vernunftnatur zu handeln und sich selbst als Teil der kosmischen Allnatur zu begreifen.

27 Seneca beschreibt, dass Menschen häufig nicht bereit seien, sich selbst als avarus, cupidus, ambitiosus, sumptuosus oder iracundus zu erkennen, sondern diese Verhaltensweisen den Umständen zuschrieben. Ohne eine solche Selbsterkenntnis könne es jedoch keine Heilung geben (epist. 50,3 et seq.).

28 Im Werk ist insgesamt die Tendenz spürbar, aus der Perspektive der Oberschicht deren Belange in den Blick zu nehmen. Die Besprechung von Fehlverhalten, das mit einem hohen Geltungsbedürfnis zusammenhängt, ist vor diesem Hintergrund also nicht überraschend.

29 Saepe enim noxia concupiscimus, nec dispicere, quam perniciosa sint, licet, quia iudicium interpellat affectus. (benef. 2,14,1). 
zentralen Gedanken der stoischen Ethik: Wie sich auch sonst im Werk immer wieder zeigt, unterscheidet Seneca zwei Ebenen einer jeden Wohltat - einmal die äußerlich sichtbare der gegebenen Sache und andererseits die der zugrundeliegenden Haltung. ${ }^{30}$ Wenn nur letztere für ihn wahrhaft von Bedeutung ist, dann deshalb, weil sie als Indikator für die innere Disposition des Handelnden gelten kann. ${ }^{31}$ Diese Betonung der Gabehaltung lenkt den eigenen Fokus von der Relevanz äußerer Gegebenheiten weg und damit zum eigenen Wesenskern hin, dessen Gestaltung (eben anders als äußere Parameter) immer in der eigenen Hand liegt. Seneca ruft etwa zu einer honesta contentio auf, die sich dann ergibt, wenn Handelnde bemüht sind, einander in ihrer Tugendhaftigkeit zu übertreffen. ${ }^{32}$

In der Vorstellung der honesta contentio ist außerdem eine erzieherische Komponente enthalten, die sich aus der Dynamik der ineinandergreifenden Haltungen ergibt und ebenfalls Bestandteil der Therapie von Undankbarkeit ist: In dem beschriebenen Wettbewerb ermutigt jeder der Handelnden durch sein Tun den anderen, noch hochherziger aufzutreten. Für den Fall, dass man es mit einem undankbaren Partner zu tun hat, kann das eigene Verhalten ihm als Orientierung dienen, selbst auch in dieser Weise aufzutreten: Hartnäckige Zuwendung soll ihn perspektivisch dankbar machen. ${ }^{33}$

Einer der möglichen Gründe, weswegen ein vormals undankbarer Partner sein Verhalten ändert, ist, dass er durch die vielfach vor Augen gestellte Tugendhaftigkeit beschämt wird (pudor). Seneca führt aus, dass Beschämung ein wirksames Mittel sein kann, um Menschen von allgemein als fehlerhaft anerkannten Verhaltensweisen abzubringen (benef. 3,16). Bestimmte Verhaltensweisen seien sogar weithin verhasst und führten denjenigen, der sie an den Tag lege, in die soziale Isolation. ${ }^{34}$

In diesen Ausführungen verfolgt Seneca eine Art Abschreckungsstrategie, indem er deutlich macht, welche unangenehmen Folgen undankbares Verhalten haben kann. Wiederum baut er auf dem Bedürfnis nach sozialer Geltung und Auszeichnung auf, welches die Handelnden leitet - und welches bewirken dürfte, dass soziale Ächtung für sie ein

30 Multum interest inter materiam beneficii et beneficium; itaque nec aurum nec argentum nec quicquam eorum, quae pro maximis accipiuntur, beneficium est, sed ipsa tribuentis voluntas. (benef. 1,5,2).

31 Diese innere Disposition des Menschen ist nach der stoischen Ethik das Einzige, worauf es ankommt; alle äußeren Güter sind an sich ohne Wert. Sie werden als adiaphora oder, lateinisch, indifferentia bezeichnet: Sie mögen angenehm sein, zählen aber nicht. Hierzu Vogt (2008b: p. 168): „Just as much as only virtue is good, only virtue benefits. According to the Stoics, valuable things do not benefit, no matter how perfectly they are selected (or used). It is thus misleading to think of benefit as some kind of ,link' between things of value and the good - as if both valuable and good things could benefit, if only the valuable things are dealt with correctly. The Stoic notion of ,benefit' is no less ambitious than the Stoic notion of good“" "Zur stoischen Würdigung der adiaphora cf. Hadot (1991: p. 79-82).

32 ... magnum ipsis certamen proponere, eos, quibus obligati sunt, re animoque non tantum aequare, sed vincere... (benef. $1,4,3) ; \ldots$ ad hanc honestissimam contentionem beneficiis beneficia vincendi... (benef. 1,4,4).

33 Qualiscumque priorum eventus est, persevera in alios conferre; melius apud ingratos iacebunt, quos aut pudor aut occasio aut imitatio aliquando gratos poterit efficere. (benef. 1,2,4).

34 Inpunita tu credis esse, quae invisa sunt, aut ullum supplicium gravius existimas publico odio? Poena est, quod non audet ab ullo beneficium accipere, quod non audet ulli dare, quod omnium designatur oculis aut designari se iudicat? (benef. 3,17,1-2). 
Risiko wäre, welches sie ungern eingehen würden; wiederum stellt er das Geltungsbedürfnis als solches nicht in Frage.

Nun müssen diejenigen, deren Verhalten Seneca in den Blick nimmt, zwar nicht grundsätzlich von ihrem Anspruch abrücken, doch sollen sie immerhin jede ihrer Handlungen prüfen, gegebenenfalls modifizieren und sich bemühen, an ihrer inneren Haltung zu arbeiten. Seneca formuliert dabei Handlungsvorschläge, die machbar erscheinen. In dieser grundsätzlichen Umsetzbarkeit zeigt sich, was Seneca im zweiten Buch De beneficiis auch selbst formuliert: Er richte sich an proficientes, an solche also, die den Versuch wagten, ihr eigenes Verhalten zu verbessern, darin aber auch immer wieder scheiterten. ${ }^{35}$ Senecas Überlegungen sind für inperfecti homines gedacht und an deren Denkhorizont orientiert.

Strenggenommen ist nur die Tugend von Belang und alles andere, Äußere, nicht der Rede wert, doch Seneca sieht sehr wohl, dass das Ideal des leidenschafts- und bedürfnislosen Weisen kaum je erreicht werden kann; umgekehrt ist er aber überzeugt, dass prinzipiell alle Menschen im Rahmen ihrer Möglichkeiten dazu imstande sind, ihren Affekten entgegenzutreten, anstatt ihnen unhinterfragt zu willfahren. ${ }^{36}$ Was Seneca seinen Adressaten in De beneficiis zumutet, ist (nur) soviel, wie für sie zunächst akzeptabel erscheint: Er drängt nicht auf eine grundsätzliche Auseinandersetzung mit ihren Ansprüchen, wohl aber darauf, dass sie die Bereitschaft zeigen, ihr Verhalten zu reflektieren. Für Seneca kommt es auf den Willen an, besser zu handeln; voluntas ist einer der Schlüsselbegriffe von De beneficiis. ${ }^{37}$

Wie der Mensch diesen Willen entwickelt, wird nicht umfassend ausgeführt. Seneca weiß, dass der Aufbruch hin zu einer Neugestaltung des eigenen Handelns schwer fällt und nicht ohne Zwang vonstatten gehen kann. ${ }^{38}$ Es sei nötig, bei denen, die falsch handeln, die Erkenntnis herbeizuführen, worauf es im Leben ankomme, denn erst dann könnten konkrete Ratschläge ( praecepta) eine heilsame Wirkung entfalten. ${ }^{39}$ Bezogen auf die besprochenen Beispiele hieße das etwa, dass die Undankbaren die Gabehaltung als

35 Totiens admoneam necesse est non loqui me de sapientibus, quos, quidquid oportet, et iuvat, qui animum in potestate habent [...], sed de inperfectis hominibus honestam viam sequi volentibus, quorum adfectus saepe contumaciter parent? (benef. 2,18,4). Hierzu Griffin (2013: p. 197). Der stoische Weise lebt in unerschütterlicher Konsistenz: quid est sapientia? semper idem velle atque idem nolle (epist. 20,5). Cf. Schofield (2003: pp. 243 et seqq.); Fuhrer (2010: p. 74); Chaumartin (1985: p. 209). Die Handlungen des Weisen wären in den Kategorien der Stoa katorthomata, vollständig richtige, wesensgemäße Taten also. Verhaltensweisen, die Nicht-Weisen nahegelegt werden, können keine katorthomata sein, wohl aber kathekonta, die dem Menschenwesen entsprechen, von relativer Richtigkeit sind, aber nicht auf dem Fundament stabiler Einsicht ausgeführt werden. Hierzu Cic. off. 1,8.

36 Dieser Optimismus durchzieht das Werk, wenngleich sich einige wenige Äußerungen finden, in denen Seneca manche Menschen als quasi unbelehrbar bezeichnet (cf. benef. 4,26 et seq.).

37 Picone (2013: p. 97), stellt heraus: „Il concetto di voluntas è di fondamentale importanza in tutta l'opera filosofica di Seneca; ancor più, nel trattato de beneficiis, il beneficio stesso viene a coincidere con la tribuentis voluntas." Setaioli (2014b: p. 285); Fuhrer (2010: bes. pp. 69-72); Inwood (2008: pp. 117 et seq.). Der Begriff voluntas ist tief in der stoischen Ethik verwurzelt; hierzu auch Bees (2004: p. 285).

38 ... initium ad illas [sc. virtutes] eundi arduum, quia hoc proprium inbecillae mentis atque aegrae est, formidare inexperta; itaque cogenda est ut incipiat. (epist. 50,9).

39 Nihil enim proficient praecepta quamdiu menti error obfusus est: si ille discutitur, apparebit quid cuique debeatur officio. Alioqui doces illum, quid sano faciendum sit, non efficis sanum. (epist. 94,5). 
dem Gabeinhalt überlegen begreifen, um sodann den Rat beherzigen zu können, dass es auf deren Gestaltung ankommt. Besser zu handeln meint also zugleich immer auch besser zu werden.

In der Funktion des Unterweisenden - der also die einzelnen Fehlhaltungen erkennt und Wege zu deren Korrektur weist - erscheint Seneca im Wortsinn als Therapeut, dessen Aufgabe darin besteht, kranke Seelen gründlich zu behandeln. ${ }^{40}$ Zugleich ist im vierten Buch De beneficiis die - genuin stoische - Überzeugung greifbar, ${ }^{41}$ dass jedem Menschen das Wissen um das Gute, Tugendhafte innewohne; ${ }^{42}$ damit müsste an sich jeder in der Lage sein, aus eigener Kraft zu einer Besserung zu finden. In dieser Überzeugung gründet die am Ende des Werkes skizzierte Empfehlung einer cotidiana meditatio, die denjenigen, der sie betreibe, allmählich zu seelischer Gesundheit führe. ${ }^{43}$

Die Selbstbetrachtung ist dazu geeignet, die eigenen Fehlhaltungen zu erkennen und, indem man sich von ihnen befreit, ein besserer Mensch zu werden. ${ }^{44}$ Konsequent stoisch gedacht, können Menschen aus Einsicht oder innerer Not heraus die Entscheidung treffen, ihr eigenes Handeln stärker reflektieren zu wollen, und sich also allein auf den Weg der Seelenheilung begeben. In diesem Sinne ist Senecas philosophisches Werk nicht nur Unterweisung (anderer), sondern auch Selbstheilung, denn die Tatsache, dass er selbst philosophisch tätig ist, nimmt ihn aus dem Kreis der zu Therapierenden nicht aus. Wenngleich er - aufgrund des relativen Vorsprungs, den er hat, indem er um ethische Leitprinzipien weiß - anleitend und auch behandelnd wirken kann, ist die Therapie letztendlich doch eine Aufgabe, der sich jeder Einzelne - soweit er den Willen dazu entwickelt - selbst stellen muss. ${ }^{45}$

Sowohl in Bezug auf die Unterweisung als auch auf die Selbstbetrachtung wird von absolut richtigen Prinzipien ausgegangen, deren Erkenntnis notwendig ist, um Fehlhaltungen

40 Itaque debemus [...] percurare mentem aegram. (epist. 94,13).

41 Diese Überzeugung wurzelt in der stoischen Oikeiosislehre, nach der alle Lebewesen instinkthaft die eigenen „characteristic features“ (Begriff nach Klein 2016: p. 171) erfassen, welche eine je spezifische Form artgemäßen Lebens zur Folge haben. Was den Menschen betrifft, so zeichnet ihn einerseits seine Vernunftbegabtheit aus, andererseits seine Geselligkeit (benef. 4,18,1). Zu Verhaltensweisen, die seinem Wesen entsprechen, zieht es ihn hin (epist. 50,8).

42 ... adeoque gratiosa virtus est, ut insitum sit etiam malis probare meliora (benef. 4,17,2).

43 Prägnant: ... eoque cotidiana meditatione perduci, ut sua sponte occurrant salutaria et ubique ac statim desiderata praesto sint et sine ulla mora veniat illa turpis honestique distinctio. (benef. 7,2,1).

44 Seneca erkundigt sich in epist. 50,1 nach dem Befinden seines Adressaten Lucilius und stellt die Frage, womit er seine Zeit verbringe. Dann vermutet er: Quid enim aliud agis quam ut meliorem te ipse cotidie facias, ut aliquid ex erroribus ponas, ut intellegas tua vitia esse quae putas rerum? Lucilius macht sich also selbst zum besseren Menschen.

45 In De beneficiis changiert Seneca zwischen der Rolle des Unterweisenden und der eines Menschen, der sich selbst der Gruppe Heilungsbedürftiger zuordnet. Bereits im Prooem wechselt er zwischen dem „nos inclusivo“ (Begriff nach Raccanelli 2009: p. 316) das ihn selbst den zu Behandelnden zurechnet, und Imperativen in der dritten Person, die ihn als denjenigen ausweisen, der anderen Verhaltensweisen nahelegt, von denen er selbst bereits weiß, dass sie richtig sind. Zu der Frage, ob jemand, der selbst kein Weiser ist, philosophische praecepta entwickeln kann, Vogt (2008a: p. 83): „The Stoics can present true claims without having either knowledge or wisdom. [...] For the purpose of working with the conception of a perfect person (or soul, or agent) it does not matter that one is not such a person." Für Hadot (1991: p. 176) ist antike Philosophie „eine Aufforderung an jeden Menschen, sich selbst umzuformen.“ 
abzulegen. Senecas Therapie der Undankbarkeit ist, dies sei abschließend angemerkt, stark normativ gedacht und bezeichnet eine den jeweiligen Möglichkeiten der proficientes angemessene Anwendung stoischer Theorie auf das eigene Handeln.

\section{Bibliographie}

\section{Primärquellen}

Préchac, F. (Ed.). (1926-1927). Sénèque: Des bienfaits. Paris: Les Belles Lettres.

Reynolds, L. D. (Ed.). (1965). L. Annaei Senecae ad Lucilium Epistulae Morales (Tomus 1). Oxford: Oxford University Press.

Winterbottom, M. (Ed.). (1994). M. Tulli Ciceronis De officiis. Oxford: University Press.

\section{Sekundärquellen}

Abel, K. (1995). Die Sinnfrage des Lebens. Philosophisches Denken im Vor- und Umfeld des frühen Christentums. Stuttgart: Steiner.

Asanger, R., \& Wenninger, G. (Eds.). (19945). Handwörterbuch Psychologie. Weinheim: Beltz.

Bees, R. (2004). Die Oikeiosislehre der Stoa, I: Rekonstruktion ihres Inhalts. Würzburg: Königshausen \& Neumann.

Chaumartin, F.-R. (1985). Le De Beneficiis de Sénèque: sa signification philosophique, politique et sociale. Paris: Les Belles Lettres.

Därmann, I. (2010). Theorien der Gabe zur Einführung. Hamburg: Junius.

Delatte, L., \& Evrard, É. et al. (Eds.). (1981). Lucius Annaeus Seneca, Opera Philosophica, Index Verborum. Hildesheim - New York: Olms.

Fuhrer, Th. (2010). Wollen oder Nicht(-)Wollen: Zum Willenskonzept bei Seneca. In J. Müller, \& R. Hofmeister Pich (Eds.), Wille und Handlung in der Philosophie der Kaiserzeit und Spätantike (pp. 69-94). Berlin - New York: De Gruyter.

Griffin, M. T. (2013). Seneca on Society. A Guide to De beneficiis. Oxford: University Press.

Hadot, P. (1991). Philosophie als Lebensform: Geistige Übungen in der Antike. Berlin: Gatza.

Inwood, B. (2008). The Will in Seneca the Younger. In J. G. Fitch (Ed.), Seneca (pp. 114-135). Oxford: Oxford University Press.

Jehne, M. (2015). From patronus to pater. The Changing Role of Patronage in the Period of Transition from Pompey to Augustus. In M. Jehne, \& F. Pina Polo (Eds.), Foreign clientelae in the Roman Empire (pp. 297-319). Stuttgart: Steiner.

Junghanß, A. (2017, i. E.). Zur Bedeutung von Wohltaten für das Gedeihen von Gemeinschaft. Cicero, Seneca und Laktanz über beneficia. Stuttgart: Steiner.

Klein, J. (2016). The Stoic Argument from Oikeiosis. Oxford Studies in Ancient Philosophy, 50, 143200.

Long, A. A. (2003). Roman philosophy. In D. Sedley (Ed.), The Cambridge Companion to Greek and Roman Philosophy (pp. 184-210). Cambridge: University Press. 
Mauss, M. (1990). Die Gabe: Form und Funktion des Austauschs in archaischen Gesellschaften. Frankfurt - Main: Suhrkamp.

Picone, G. (Ed.). (2013). Le regole del beneficio. Commento tematico a Seneca, De beneficiis, libro 1. Palermo: Palumbo.

Raccanelli, R. (2009). Cambiare il dono: per una pragmatica delle relazioni nel de beneficiis senecano. In G. Picone, L. Beltrami, \& L. Riccottilli, Benefattori e beneficati. La relazione asimmetrica nel de beneficiis di Seneca (pp. 303-356). Palermo: Palumbo.

Schofield, M. (2003). Stoic Ethics. In B. Inwood (Ed.), The Cambridge Companion to the Stoics (pp. 233-256). Cambridge: Cambridge University Press.

Setaioli, A. (2014a). Ethics I: Philosophy as Therapy, Self-Transformation, and „Lebensform“. In G. Damschen, \& A. Heil (Eds.), Brill's Companion to Seneca. Philosopher and Dramatist (pp. 239-256). Leiden - Boston: Brill.

Setaioli, A. (2014b). Ethics III: Free Will and Autonomy. In G. Damschen, \& A. Heil (Eds.), Brill's Companion to Seneca. Philosopher and Dramatist (pp. 277-299). Leiden - Boston: Brill.

VandenBos, G. R. (Ed.). (2007). APA Dictionary of Psychology. Washington: American Psychological Association.

Vogt, K. M. (2008a). Law, Reason, and the Cosmic City. Political Philosophy in the Early Stoa. Oxford: University Press.

Vogt, K. M (2008b). The Good is Benefit: On the Stoic Definition of the Good. In J. J. Cleary, \& G. M. Gurtler (Eds.), Proceedings of the Boston Area Colloquium in Ancient Philosophy (Vol. 23; pp. 155-186). Boston: Brill.

Wolkenhauer, J. (2014). Senecas Schrift De beneficiis und der Wandel im römischen Benefizienwesen. Göttingen: V\&R Unipress.

Dr. Antje Junghanß / antje.junghanss@tu-dresden.de Institute of Classical Philology / Latin Philology

Dresden University of Technology, Faculty of Linguistics, Literature and Cultural Studies

Wiener Str. 48; Raum 404, 01062 Dresden, Germany 
